\title{
Hypermethylation of tumor necrosis factor decoy receptor gene in non-small cell lung cancer
}

\author{
YUANLIN QI ${ }^{1 *}$, LIN QI $^{1 *}$, MINGLIAN QIU $^{2 *}$, CAIYUN YAO $^{1}$, MINGFANG ZHANG $^{1}$, JIANBO LIN $^{2}$, \\ ZHONGHUA ZHENG ${ }^{3}$, CHUJIA CHEN $^{3},{\text { HONGXIANG } \mathrm{LI}^{3} \text { and SHIWEI DUAN }}^{3}$ \\ ${ }^{1}$ School of Basic Medical Sciences, Fujian Medical University, Fuzhou, Fujian 350122; \\ ${ }^{2}$ Department of Thoracic Surgery, First Affiliated Hospital of Fujian Medical University, Fuzhou, Fujian 350005; \\ ${ }^{3}$ Medical Genetics Center, School of Medicine, Ningbo University, Ningbo, Zhejiang 315211, P.R. China
}

Received April 28, 2019; Accepted March 6, 2020

DOI: 10.3892/ol.2020.11565

\begin{abstract}
Abnormal methylation of the TNFRSF10C and TNFRSF10D genes has been observed in numerous types of cancer; however, no studies have investigated the methylation of these genes in non-small cell lung cancer (NSCLC). The aim of the present study was to investigate the association between TNFRSF10C and TNFRSF10D methylation and NSCLC. Methylation levels of 44 pairs of NSCLC tumor tissues and distant non-tumor tissues were analyzed using quantitative methylation specific PCR and methylation reference percentage values (PMR). The methylation levels of the TNFRSF10C gene in NSCLC tumor tissue samples were significantly higher compared with those in the distant non-tumor tissues (median PMR, $2.73 \%$ vs. $0.75 \%$; $\mathrm{P}=0.013$ ). Subgroup analysis demonstrated that the methylation levels of TNFRSF10C in tumor tissues from male patients were significantly higher compared with those in distant non-tumor tissues (median PMR, $2.73 \%$ vs. $0.75 \%$; $\mathrm{P}=0.041$ ). The levels of TNFRSF10C methylation were also higher in the tumor tissues of patients who were non-smokers compared with their distant non-tumor tissues (median PMR, $2.50 \%$ vs. $0.63 \%$; $\mathrm{P}=0.013)$. TNFRSF10C methylation levels were higher in the tumor tissues from male patients compared with those from female patients (median PMR, $2.50 \%$ vs. $0.63 \%$; $\mathrm{P}=0.031$ ). However, no significant differences in the methylation levels
\end{abstract}

Correspondence to: Dr Yuanlin Qi, School of Basic Medical Sciences, Fujian Medical University, 1 Xuefu North Road, Fuzhou, Fujian 350122, P.R. China

E-mail: ylqi@fjmu.edu.cn

Dr Shiwei Duan, Medical Genetics Center, School of Medicine, Ningbo University, 818 Fenghua Road, Ningbo, Zhejiang 315211, P.R. China

E-mail: duanshiwei@nbu.edu.cn

${ }^{*}$ Contributed equally

Key words: non-small cell lung cancer, gene methylation, TNFRSF10C, TNFRSF10D, promoter of the TNFRSF10D gene were observed between the sexes. Using the cBioPortal and The Cancer Genome Atlas lung cancer data, it was demonstrated that TNFRSF10C methylation levels were inversely correlated with TNFRSF10C mRNA expression levels ( $\mathrm{r}=-0.379 ; \mathrm{P}=0.008)$. In addition, demethylation of lung cancer cell lines A549 and NCI-H1299 using 5'-aza-deoxycytidine further confirmed that TNFRSF10C hypomethylation was associated with significant upregulation of TNFRSF10C mRNA expression levels [A549 fold-change $(\mathrm{FC})=8 ; \mathrm{P}=1.0 \times 10^{-4}$; NCI-H1299 $\mathrm{FC}=3.163$; $\left.\mathrm{P}=1.143 \times 10^{-5}\right]$. A dual luciferase reporter gene assay was also performed with the insert of TNFRSF10C promoter region, and the results revealed that the TNFRSF10C gene fragment significantly enhanced the transcriptional activity of the reporter gene compared with that in the control group $(\mathrm{FC}=1.570 ; \mathrm{P}=0.032)$. Overall, the results of the present study demonstrated that hypermethylation of TNFRSF10C was associated with NSCLC.

\section{Introduction}

Lung cancer is the leading cause of cancer-associated mortality among males and the second leading cause of cancer-associated mortality among females globally, with an estimated 1.8 million new cases, and 1.6 million mortalities in 2012 (1). In the past 30 years, the number lung cancer-associated deaths in China has increased by $464.84 \%$, and $\sim 600,000$ individuals die of lung cancer each year (2). In recent years, with the advances in early diagnosis and clinical treatment techniques, lung cancer treatment and diagnosis has improved (3). However, the number of patients with lung cancer in China has grown at a rate of $0.9 \%$ per year between 2000 and 2011 (2). Due to China's large population, such a growth rate causes a huge economic burden on public health.

Non-small cell lung cancer (NSCLC) includes large cell lung cancer, adenocarcinoma and squamous cell carcinoma, accounting for $\leq 85 \%$ of lung cancer cases (4). For NSCLC, surgery is the best treatment (5). However, only $25-30 \%$ of patients are suitable for potentially curative resection (5). At present, the 5-year survival rates of patients with stage Ia and IIIa NSCLC undergoing resection are 75 and $25 \%$, respectively (5). Therefore, the development of new clinical 
treatments and methods of early diagnosis for lung cancer is essential.

The occurrence of lung cancer is associated with smoking, radon, air pollution and occupational factors like asbestos. Recently, several studies have reported that the process of carcinogenesis is driven by the accumulation of genetic mutation, such as K-Ras and EGFR, as well as epigenetic changes (6-8). Epigenetic changes include DNA methylation, histone modifications and expression of non-coding RNA (9). Tumor development is primarily associated with the inactivation of tumor suppressor genes and the activation of oncogenes (8). DNA methylation in the promoter region can prevent the activation of a gene, leading to a downregulation of its expression (10). Previous studies have indicated that the primary markers of lung cancer include the inactivation of various tumor suppressor gene promoters by methylation. For example, aberrantly methylated genes associated with homeoboxes are frequently observed in early stage lung cancer, including SIX homeobox (SIX), LIM homeobox (LHX), paired box (PAX) and distal-less homeobox (DLX) (11). Hypermethylated tumor suppressor genes commonly found in advanced non-small cell lung cancer include: Helicase-like transcription factor (HLTF), adenovirus E1B $19 \mathrm{kDa}$ interacting protein 3 (BNIP3), member $X$ in H2A histone family $(H 2 A F X)$, calcium voltage-gated channel subunit alphal $G$ (CACNAIG), TGFB-induced factor homebox 1 (TGIF), inhibitor of DNA binding 4 (ID4) and calcium voltage-gated channel subunit alphal A (CACNAIA) (12). The present study aimed to identify aberrantly methylated genes in tumors, and then to develop diagnostic markers for NSCLC in plasma or other body fluids.

Tumor necrosis factor (TNF) receptor superfamily member 10c (TNFRSF10C) and TNFRSF10D are decoy receptors for the TNF-related apoptosis-inducing ligand (TRAIL), a member of the TNF family (13). TNFRSF10C and TNFRSF10D bind to TRAIL and do not contain the death domain necessary for apoptosis; thus, they inhibit TRAIL-induced apoptosis by competing with the TRAIL receptors (TRAIL-R) death receptor 4 (DR4) and DR5 for binding TRAIL (13). TRAIL and its associated receptors have been used as targets for anticancer therapeutics (14). The tolerability and safety of TRAIL was determined in clinical phase I studies, and no dose-limiting toxicity was observed $(15,16)$. TRAIL-R agonists can be used as a single agent or in combination with conventional chemotherapy drugs for advanced NSCLC (17), colorectal cancer (18), B-cell lymphoma (19) and advanced or metastatic solid tumors or non-Hodgkin's lymphoma (NHL) (20). TRAIL ligands are widely expressed in tumor tissues and have attracted attention due to their ability to selectively induce tumor cell apoptosis (21).

The methylation levels of TNFRSF10C and TNFRSF10D in lung cancer have been studied in lung adenocarcinoma (22). A previous study explored tumor-associated genes, in which TNFRSF10C methylation levels were higher in patients with lung adenocarcinoma who were non-smokers compared with those who were smokers (22). However, the aforementioned study did not compare the methylation levels of TNFRSF10C and TNFRSF10D genes between lung cancer and normal tissues. In the present study, the association between TNFRSF10C and TNFRSF10D methylation levels and NSCLC was investigated by analyzing NSCLC and distant non-tumor tissues from patients from The First Affiliated Hospital of Fujian Medical University (Fuzhou, China) and The Cancer Genome Atlas (TCGA) database. The aim of the present study was to identify the contribution of methylation of the aforementioned genes to NSCLC pathogenesis.

\section{Materials and methods}

Tissue sample collection. A total of 44 pairs of tumor tissues and distant non-tumor tissues were collected from June 2017 to September 2018. These samples were obtained from 44 patients with NSCLC at The First Affiliated Hospital of Fujian Medical University (Fuzhou, China). Patient clinicopathological information was collected, including age, sex, family history. The average age of the patients was 62.91 \pm 9.05 years (mean \pm SD; age range, $42-87$ years), including 23 female and 21 male patients. There were 32 cases of adenocarcinoma, 12 cases of squamous cell carcinoma and 2 inflammatory pseudotumors. The research protocol was approved by The Ethics Committee of the First Affiliated Hospital of Fujian Medical University (Fuzhou, China; approval number, 2017-KY-068). All subjects provided written informed consent. Samples were taken during resection and the sampling procedure was performed under the guidance of a pathologist to distinguish between tumor tissues and distant non-tumor tissues ( $>10 \mathrm{~cm}$ away from the lesions).

Quantitative methylation-specific PCR (qMSP) assay. Primer sequences were designed based on the region of the TNFRSF10C and TNFRSF10D genes rich in CG sites. The primer sequences were as follows: TNFRSF10C forward, 5'-AGGGTGCGATTTAGGATTTAG-3' and reverse, 5'-CGA TAACGACGACGAACTT5'; TNFRSF10D forward, 5'-CGA CGATGAAGACGACGAAT-3' and reverse, 5'-AAACCAAAC CATAACTCCTAAACC-3'. The conditions for qMSP were as follows: Initial denaturation at $95^{\circ} \mathrm{C}$ for $10 \mathrm{~min}$, denaturation at $95^{\circ} \mathrm{C}$ for $20 \mathrm{sec}$, annealing at $56^{\circ} \mathrm{C}$ for $20 \mathrm{sec}$ and extension at $72^{\circ} \mathrm{C}$ for $30 \mathrm{sec}$ for 45 cycles. The melting curve program included $95^{\circ} \mathrm{C}$ for $15 \mathrm{sec}, 60^{\circ} \mathrm{C}$ for $60 \mathrm{sec}$ and then from $60^{\circ} \mathrm{C}$ to $95^{\circ} \mathrm{C}$ at a speed of $0.11^{\circ} \mathrm{C} / \mathrm{sec}$.

DNA was extracted from patient tissue samples according to the instructions of the QIAamp DNA FFPE Tissue kit (Qiagen $\mathrm{GmbH}$ ). The extracted samples were measured for purity and concentration using a NanoDrop 1000 spectrophotometer (Thermo Fisher Scientific, Inc.). The DNA concentration of all samples was $>25 \mathrm{ng} / \mu \mathrm{l}$, and the purity of DNA was 1.8-2.0 based on the absorbance ratio at 260 and $280 \mathrm{~nm}$. The DNA was subjected to bisulfite modification using the EZ DNA Methylation-Gold ${ }^{\mathrm{TM}}$ kit according to the manufacturer's instructions (Zymo Research Corp.). The primers and the bisulfite-modified DNA were then subjected to qMSP. The total reaction system was $10 \mu \mathrm{l}$, containing $5 \mu \mathrm{l} \mathrm{SYBR}$ mixture, $4 \mu \mathrm{l}$ double-distilled $\mathrm{H}_{2} \mathrm{O}, 0.5 \mu \mathrm{l}$ each of the forward and reverse primers and $0.5 \mu \mathrm{l}$ modified DNA. To avoid errors in sample loading, $A C T B$ was used as an internal reference for each sample. The primer sequences of ACTB were as follows: Forward, 5'-TGGTGATGGAGGAGGTTTAGTAAGT-3' and reverse, 5'-AACCAATAAAACCTACTCCTCCСТTAA-3'. A total of $100 \%$ M-Sss I (New England BioLabs, Inc.) treated sperm DNA was used as the positive control and nuclease-free 
water was used as the negative control for each group. The qMSP product was then subjected to Qsep100 DNA fragment analysis (BiOptic, Inc.) and visualized. Three randomly picked qMSP products of each gene were Sanger sequenced for validation.

$A C T B$ is a housekeeping gene that is often stably expressed in most healthy and tumor cells; thus, it is commonly used as an internal reference for quantitation of mRNA expression levels and qMSP assays (23). Completely methylated DNA referred to human sperm DNA treated with methylated-SssI, which allowed all $\mathrm{C}$ sites in the DNA to be methylated, including CG sites, and thus was used as a positive control. The formula for quantifying methylation levels was used to calculate the relative methylation level by the comparison between the target gene and the $A C T B$ gene $(24,25)$. For each sample, the relative methylation value was determined using the $2^{-\Delta \Delta \mathrm{Cq}}$ method. The sample methylation reference percentage (PMR) was calculated using the following formula: $\mathrm{PMR}=2^{-\Delta \Delta \mathrm{Cq}} \times 100 \%$; $\Delta \Delta \mathrm{Cq}=$ sample DNA $\left(\mathrm{Cq}_{\text {gene }}-\mathrm{Cq}_{\mathrm{ACTB}}\right)$-fully methylated DNA $\left(\mathrm{Cq}_{\text {gene }}-\mathrm{Cq}_{\mathrm{ACTB}}\right)$.

Luciferase reporter gene assay. A recombinant plasmid pGL3-TNFRSF10C containing the selected fragment (-121 to +363 bp of TNFRSF10C, $485 \mathrm{bp}$ ) was constructed. The target plasmid pGL3-TNFRSF10C was co-transfected with the pRL-SV40 plasmid carrying the Renilla luciferase gene to provide an internal control. Cells co-transfected with the empty pGL3-Basic plasmid and the pRL-SV40 plasmid were used as negative references in each experiment. The pGL3-Promoter plasmid and the pRL-SV40 plasmid were also co-transfected as a positive control each time. The plasmids were separately transferred into $293 \mathrm{~T}$ cells $\left(10 \% \mathrm{FBS}, 37^{\circ} \mathrm{C}, 5 \% \mathrm{CO}_{2}\right.$; China Center for Type Culture Collection) using FuGENE ${ }^{\circledR}$ HD Transfection Reagent (Promega Corporation), according to the manufacturer's protocol. The target plasmid $(1 \mu \mathrm{g})$ and the Renilla luciferase plasmid $(0.1 \mu \mathrm{g})$ were transfected into $293 \mathrm{~T}$ cells at a ratio of 10:1 using FuGENE ${ }^{\circledR}$ HD. After $24 \mathrm{~h}$ of transfection, luciferase activity was detected using a dual luciferase assay kit (Promega Corporation), according to the manufacturer's protocol. The luciferase reaction intensity was measured at a wavelength of $\sim 560 \mathrm{~nm}$, using a SpectraMax 190 microplate reader (Molecular Devices) by adding the Luciferase Assay Reagent II. After the fluorescence reaction intensity was measured, the Stop\&Glo Reagent was added to the same sample to quench the aforementioned reaction, and the Renilla luciferase reaction was simultaneously initiated for the second measurement. Luciferase activity was finally calculated using the Dual Luciferase Reporter Assay system (Promega Corporation) and normalized to Renilla luciferase activity.

Cell culture and gene expression detection. The embryonic cells A549 and NCI-H1299 were purchased from China Center for Type Culture Collection, and the cells were treated with $5 \mu \mathrm{mol} / 1$ 5'-azadeoxycytidine (5-Aza; A3656, Sigma-Aldrich; Merck $\mathrm{KGaA}$ ) for $24 \mathrm{~h}$ at $37^{\circ} \mathrm{C}(25)$. RNA was extracted by TRIzol $^{\circledR}$ (Invitrogen; Thermo Fisher Scientific, Inc.) before and after 5-Aza treatment, and cDNA was obtained by using a PrimeScript RT kit (Takara Biotechnology Co., Ltd.). The quantitative PCR primers sequences (Sangon Biotech Co.,
Ltd.) were as follows: TNFRSF10C forward, 5'-TGCACA GAGGGTGTGGATTAC-3' and reverse, 5'-ATTCCGGAA GGTGCCTTCTTT-3'; ACTB forward, 5'-AGCACAGAGCCT CGCCTTT-3' and reverse, 5'-AGGGTGAGGATGCCTCTC TT-3'. The Real-time PCR system was configured using the Takara TB Green kit (Takara Biotechnology Co., Ltd.), and the mRNA expression levels of TNFRSF10C were detected using a StepOne Plus real-time PCR instrument (Applied Biosystems; Thermo Fisher Scientific, Inc.). The thermocycling conditions for qPCR were as follows: Initial denaturation at $95^{\circ} \mathrm{C}$ for $10 \mathrm{~min}, 45$ cycles of denaturation at $95^{\circ} \mathrm{C}$ for $20 \mathrm{sec}$, annealing at $56^{\circ} \mathrm{C}$ for $20 \mathrm{sec}$, extension at $72^{\circ} \mathrm{C}$ for $30 \mathrm{sec}$ and a final extension at $72^{\circ} \mathrm{C}$ for $10 \mathrm{~min}$. Relative expression levels were calculated using the $2^{-\Delta \Delta \mathrm{Cq}}$ method (25) and normalized to internal reference gene $\beta$-actin. A negative reference was also prepared using nuclease-free water.

Bioinformatics analysis. A total of 312 pairs of lung cancer tumor tissues and distant non-tumor tissues were obtained from TCGA database (https://tcga.xenahubs.net) for the analysis of differential TNFRSF10C and TNFRSF10D gene methylation levels. The correlation between methylation and mRNA expression levels of the TNFRSF10C gene (49 samples) were analyzed and charted using cBioPortal (http://www.cbioportal. org). TNFRSF10C DNA methylation and TNFRSF10C expression data from the lung adenocarcinoma (LUAD) project were obtained by downloading the data of 568 TCGA lung cancer samples from National Cancer Institute GDC Data Portal (https://portal.gdc.cancer.gov/repository).

Statistical analysis. Statistical analysis was performed using SPSS version 20.0 (IBM Corp). Nonparametric Wilcoxon signed-rank tests were used to determine the differences in methylation between tumor tissues and distant non-tumor tissues. The methylation level is expressed as the median. The nonparametric Wilcoxon rank-sum test was used to analyze the methylation differences between tumor tissues and distant non-tumor tissues in TCGA database. The correlation between TNFRSF10C methylation levels and mRNA expression levels was determined using Spearman's test. Luciferase reporter gene activities were analyzed using the Kruskal Wallis test with Dunn's post hoc test. $\mathrm{P}<0.05$ was considered to indicate a statistically significant difference.

\section{Results}

Selected promoter fragment in the current methylation assay. Corresponding primers for the CG-rich region of TNFRSF10C and TNFRSF10D were selected for analysis (Fig. 1A). The target fragments of both genes were detected using capillary electrophoresis amplification, and the amplified fragment was confirmed using Sanger sequencing (Fig. 1B and C). There were 7 and 15 cytosines (including $5 \mathrm{CG}$ and $1 \mathrm{CG}$ ) in the primer sequences of TNFRSF10C and TNFRSF10D, respectively. The qMSP melting curves indicated that the qMSP products of both genes were homogeneous (data not shown).

Differences in TNFRSF10C methylation levels in tumor tissues and distant non-tumor tissues. Methylation levels of the TNFRSF10C and TNFRSF10D genes between tumor and 
A

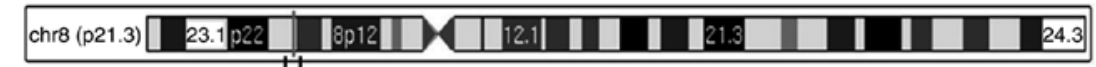
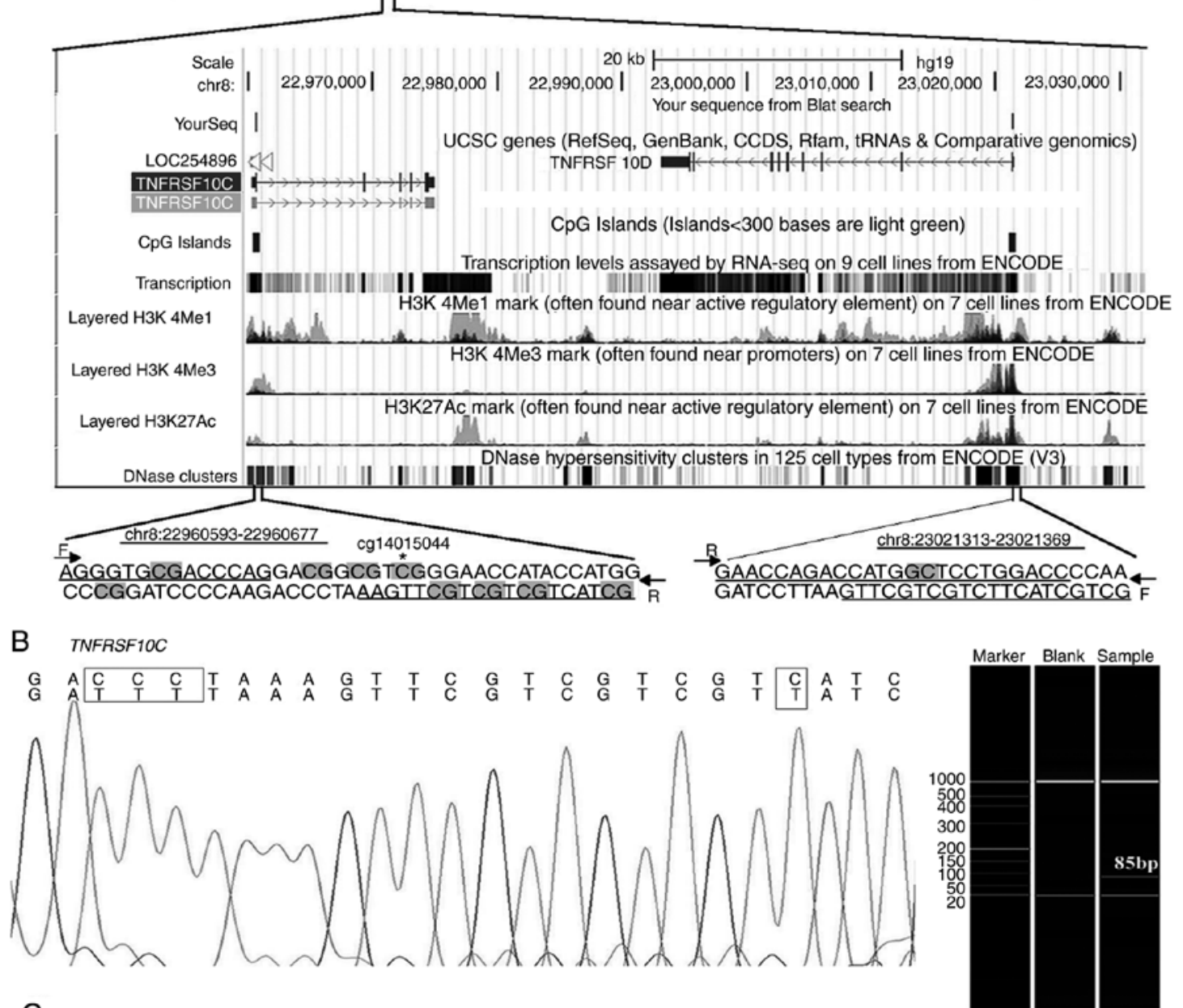

C TNFRSF10D
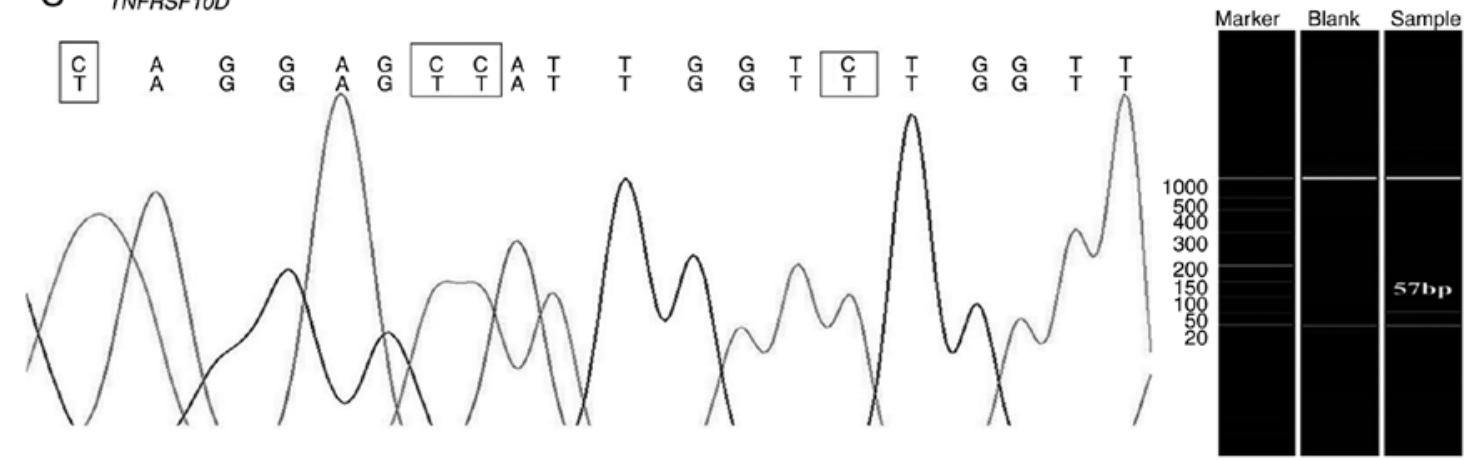

Figure 1. The genomic locations of TNFRSF10C and TNFRSF10D and the validation of qMSP products. (A) TNFRSF10C and TNFRSF10D are located at chr8p22-8p23, and the two gene segments in qMSP are located on the CG island. (B and C) Sanger sequencing results for (B) TNFRSF10C and (C) TNFRSF10D qMSP products. The upper line is the original sequence, and the lower line is the bisulfite converted sequence. Capillary electrophoresis results (right) confirmed that the sizes of the amplified products were as expected. qMSP, quantitative methylation-specific PCR; chr, chromosome; RefSeq; CCDS; Rfam; RNA-seq, RNA-sequencing; tRNAs, transfer RNAs; bp, base pair; TNFRSF, tumor necrosis factor receptor superfamily member; ENCODE, The Encyclopedia of DNA Elements.

distant non-tumor tissues were compared in 44 patients with NSCLC. The results demonstrated that the methylation levels of TNFRSF10C in tumor tissues were significantly higher compared with those in distant non-tumor tissues $(\mathrm{P}=0.013$; Fig. 2A). Further subgroup analysis of the clinicopathological data revealed a significant association between TNFRSF10C methylation levels and NSCLC in male patients and in non-smokers (Table I). In male patients, the methylation levels of TNFRSF10C in tumor tissues were higher compared with those in distant non-tumor tissues ( $\mathrm{P}=0.041$; Fig. $2 \mathrm{~B})$, and the methylation levels in tumor tissues from male patients were significantly higher compared with those in tumor tissues from female patients $(\mathrm{P}=0.013$; Fig. $2 \mathrm{~B})$. In patients who were non-smokers, TNFRSF10C methylation levels were higher in tumor tissues compared with distant non-tumor tissues $(\mathrm{P}=0.031$; Fig. 2C). However, in female patients who were non-smokers there were no significant differences in the DNA methylation levels of TNFRSF1OC between tumor tissues 
A
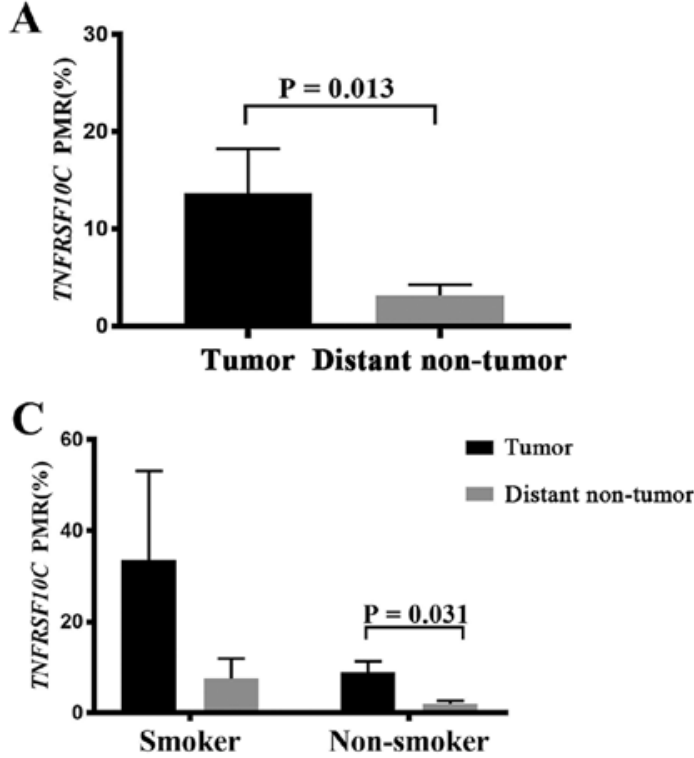

B

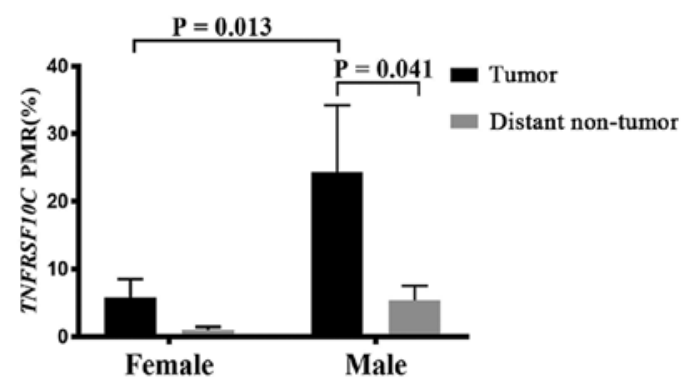

D

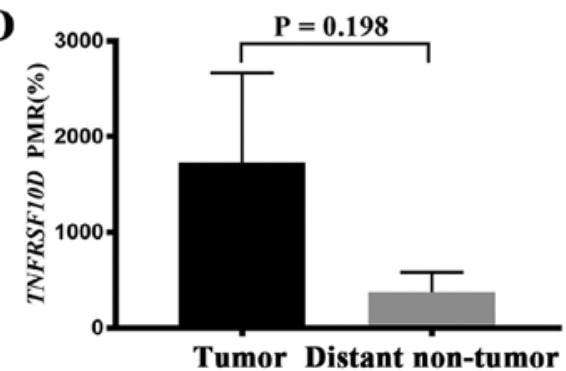

Figure 2. Differences in TNFRSF10C/TNFRSF10D methylation levels in tumor tissues and distant non-tumor tissues. (A) Differences in the level of methylation of TNFRSF10C in tumor and distant non-tumor tissues. (B) Difference in methylation levels of TNFRSF10C in tumor tissues between male and female patients. (C) Difference between TNFRSF10C methylation in NSCLC tissues and distant non-tumor tissues was significant for non-smokers. (D) Differences in the levels of methylation of TNFRSF10D in tumor tissues and distant non-tumor tissues. PMR, methylation reference percentage; TNFRSF10C, tumor necrosis factor receptor superfamily member 10c; TNFRSF10D, tumor necrosis factor receptor superfamily member 10d.

and distant non-tumor tissues $(\mathrm{P}=0.46$; Table $\mathrm{I})$. In male patients who were non-smokers, the methylation levels in tumor tissues were significantly lower compared with those in distant non-tumor tissues $(\mathrm{P}=0.03$; Table I). In male patients who were smokers, no significant difference was observed in the TNFRSF10C DNA methylation levels between tumor tissues and distant non-tumor tissues $(\mathrm{P}=0.225$; Table I). These results indicated that the association of TNFRSF10C hypomethylation with lung cancer was only observed in male patients who were non-smokers. Due to the limited number of patients in each subgroup, this conclusion needs to be interpreted with caution.

The levels of methylation of TNFRSF10D were not significantly different in the paired samples $(\mathrm{P}=0.198$; Fig. 2D). Furthermore, subgroup analysis of the clinicopathological data did not reveal any significant associations between TNFRSF10D methylation levels and NSCLC.

To ensure that the data of the inflammatory pseudotumor did not affect the results of the experiment, the data from the two inflammatory pseudotumors were removed from analysis.

TNFRSF10C methylation and expression levels. TCGA database was used to obtained methylation data for the TNFRSF10C promoter region in lung tumor tissue samples and to compare the methylation levels of TNFRSF10C between tumor tissues and distant non-tumor tissues. The results revealed that methylation levels at one of the CG sites in the TNFRSF10C promoter region was higher in tumor tissues compared with in distant non-tumor tissues samples $(\operatorname{cg} 14015044, \mathrm{P}=0.045$; Fig. 3A). Using lung cancer data from the cBioPortal database, it was reported that the methylation levels of TNFRSF10C were negatively correlated with TNFRSF10C mRNA expression levels ( $\mathrm{r}=-0.379, \mathrm{P}=0.008$; Fig. 3B). The prognostic data from male patients with lung cancer in TCGA database revealed that the TNFRSF10C methylation levels had no effect on overall patient survival time (data not shown).

TCGA data analysis also failed to identify any significant association between TNFRSF10D and lung cancer (data not shown). Overall, these results suggested no association between TNFRSF10D methylation levels and lung cancer.

Clinicopathological, TNFRSF10C DNA methylation and TNFRSF10C expression data from the lung adenocarcinoma (LUAD) project were downloaded from TCGA database. From the gene promoter region $(-2,000$ to $+500 \mathrm{bp}), 10 \mathrm{CG}$ sites closest to the qMSP amplicon presented in Fig. 4A were selected, and between-group comparison and association analyses were performed. Based on the comparison between cancer and non-cancerous tissues, it was demonstrated that multiple CG sites, such as cg08309809, were significantly associated with LUAD (Fig. 4B). Further subgroup analysis revealed an association between $\operatorname{cg} 08309809$ and LUAD in male patients (Fig. 4C), female patients (Fig. 4D) and patients who were smokers (Fig. 4E). There was no significant association between cg08309809 and LUAD in the non-smoking group (Fig. 4F); however, this may be due to the small number of non-tumor tissues $(\mathrm{n}=2)$. The methylation and expression levels of multiple CG sites in the TNFRSF10C gene were significantly inversely correlated $\left(\mathrm{r}=-0.408 \sim-0.146 ; \mathrm{P}=1.00 \times 10^{-13} \sim 2.65 \times 10^{-4}\right.$ for different CG sites; Fig. 4G), suggesting that TNFRSF10C promoter methylation may be associated with gene silencing.

Dual luciferase assay to detect promoter activity of TNFRSF10C. A dual luciferase reporter plasmid containing the promoter fragment of TNFRSF10C (-121 to $+363 \mathrm{bp})$ was constructed. The results demonstrated that the inserted gene fragment significantly enhanced the transcriptional activity of the reporter gene compared with the control group [fold-change $(\mathrm{FC})=1.570, \mathrm{P}=0.032$; Fig. 5A]. The purpose 
Table I. Association between tumor necrosis factor receptor superfamily member 10c methylation levels and clinicopathological characteristics of patients with non-small cell lung cancer.

\begin{tabular}{|c|c|c|c|c|}
\hline Variable & $\mathrm{n}$ & $\begin{array}{l}\text { Tumor PMR, median \% } \\
\text { (interquartile range) }\end{array}$ & $\begin{array}{c}\text { Distant non-tumor PMR, } \\
\text { median } \% \text { (interquartile range) }\end{array}$ & P-value \\
\hline \multicolumn{5}{|l|}{ Age } \\
\hline$\leq 60$ & 16 & $1.430(0,8.360)$ & $1.805(0.016,5.475)$ & 0.594 \\
\hline$>60$ & 28 & $4.095(0,23.650)$ & $0.459(0,2.53)$ & 0.060 \\
\hline \multicolumn{5}{|l|}{ Sex } \\
\hline Female & 23 & $0.342(0,4.970)$ & $0.105(0,1.505)$ & 0.460 \\
\hline Male & 11 & $9.690(1.365,28.645)$ & $2.535(0.081,5.925)$ & $0.041^{\mathrm{a}}$ \\
\hline \multicolumn{5}{|c|}{ Smoking history } \\
\hline Non-smoker & 34 & $2.500(0,10.330)$ & $0.639(0,2.272)$ & $0.031^{\mathrm{a}}$ \\
\hline Smoker & 10 & $2.730(0,121.05)$ & $5.640(0,8.110)$ & 0.225 \\
\hline \multicolumn{5}{|c|}{ Histological type } \\
\hline LUSC & 30 & $1.040(0,9.690)$ & $0.490(0,2.142)$ & 0.128 \\
\hline LUAD & 12 & $7.250(0,107.300)$ & $5.640(0.965,12.290)$ & 0.086 \\
\hline \multicolumn{5}{|c|}{ Cancer location } \\
\hline Right lung & 26 & $2.730(0,9.025)$ & $1.129(0,3.090)$ & 0.088 \\
\hline Left lung & 18 & $3.975(0.535,24.725)$ & $0.521(0,3.930)$ & 0.064 \\
\hline
\end{tabular}

${ }^{a} \mathrm{P}<0.05$, Wilcoxon signed-rank test. The methylation level is expressed as the median (quartile). PMR, methylation reference percentage; LUSC, lung squamous cell carcinoma; LUAD, lung adenocarcinoma.
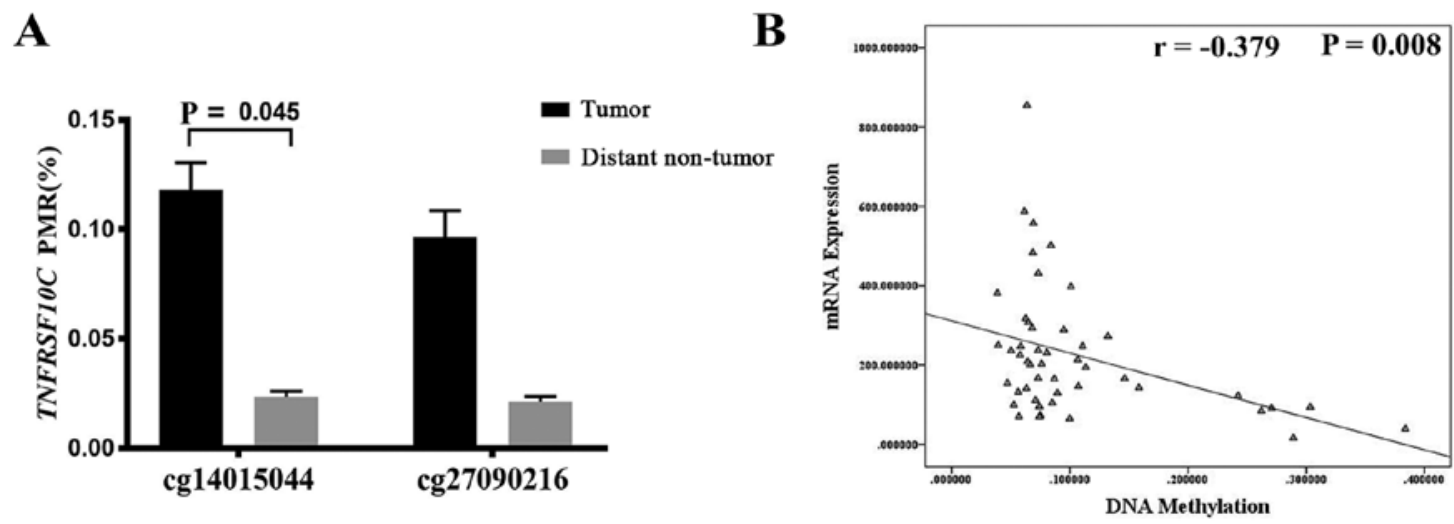

Figure 3. TNFRSF10C methylation and expression levels. (A) Differences in the methylation of CG sites in the promoter of TNFRSF10C between tumor tissues and distant non-tumor tissues. (B) TNFRSF10C methylation and mRNA expression levels were inversely correlated based on the data of 49 The Cancer Genome Atlas lung cancer cases from the cBioPortal database.

of the luciferase experiment was to confirm whether the promoter fragment regulated gene expression levels. By cloning the fragment into the reporter vector, it was demonstrated that the fragment enhanced the expression levels of the reporter gene, suggesting that the fragment regulated gene expression levels. Therefore, the methylation of this fragment may serve a role in regulating the TNFRSF10C gene expression levels. To determine the relationship between TNFRSF10C promoter methylation and its transcriptional expression levels in lung cancer cells, 5'-aza-deoxycytidine was used to treat lung cancer cells A549 and NCI-H1299. The results demonstrated that the mRNA expression levels of TNFRSF10C in the two lung cancer cell lines were significantly increased following demethylation treatment (A549,
$\mathrm{FC}=8.000, \mathrm{P}=0.0001 ; \mathrm{NCI}-\mathrm{H} 1299, \mathrm{FC}=3.163, \mathrm{P}=1.143 \times 10^{-5}$; Fig. 5B).

\section{Discussion}

Decreased gene expression levels of TRAIL decoy receptor gene TNFRSF10C caused by hypermethylation have been observed in glioblastoma (26), prostate (27) and breast (28) cancer. The results of the present study also demonstrated that the levels of methylation of TNFRSF10C in NSCLC tissues were higher compared with those in distant non-tumor tissues, which was consistent with the aforementioned studies. The present study also demonstrated that hypermethylation of TNFRSF10C was specific to tumor tissues in male patients 

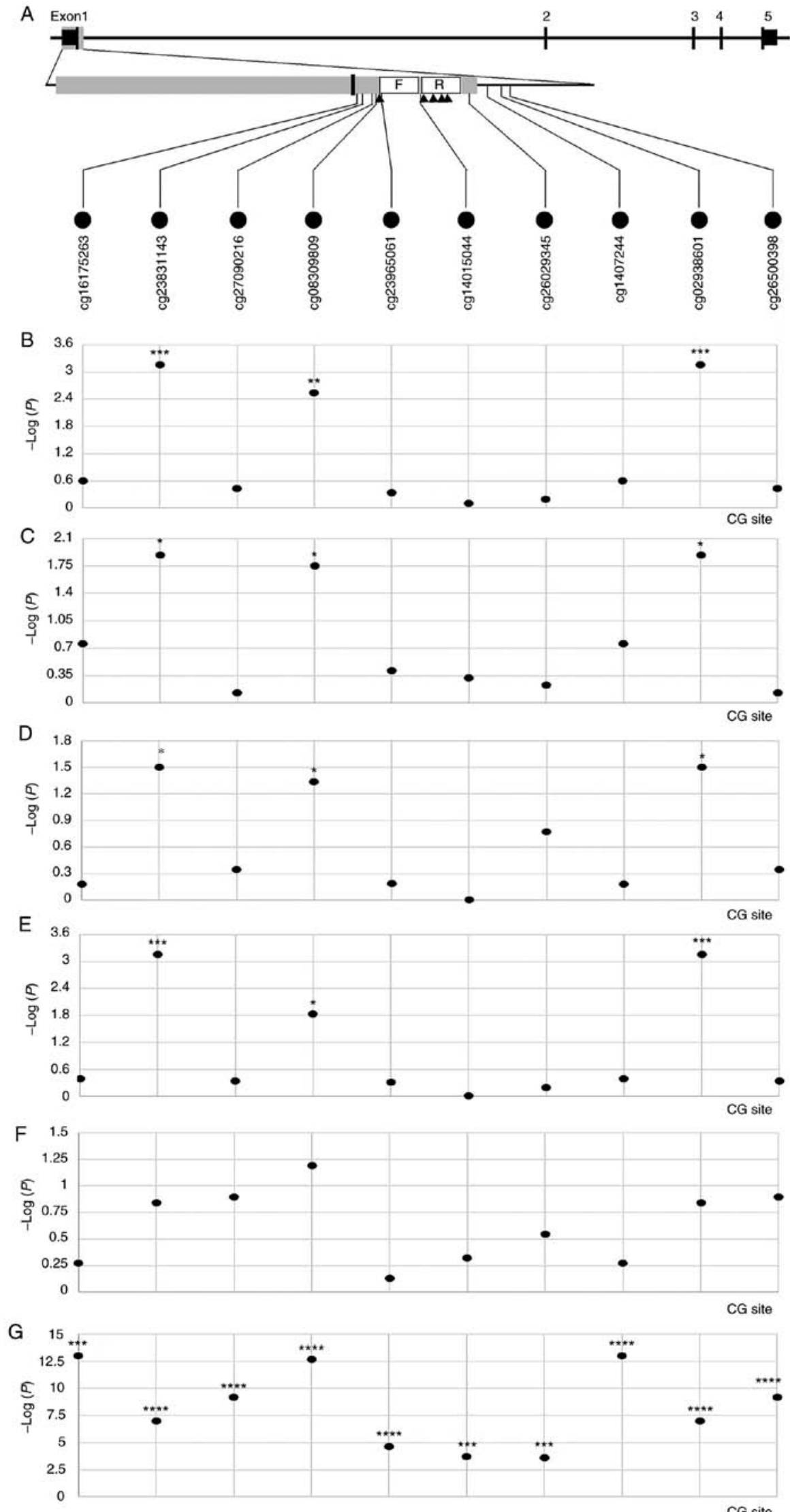

Figure 4. The Cancer Genome Atlas analysis of the association between TNFRSF10C methylation levels, lung adenocarcinoma and TNFRSF10C gene expression. (A) TNFRSF10C gene structure. $\mathbf{\Delta}$, testing CG sites. (B) Differences in the methylation levels of CG sites between tumor and para-tumor. (C) Differences in the methylation levels of CG sites between tumor and para-tumor in male patients. (D) Differences in the methylation levels of CG sites between tumor and para-tumor in female patients. (E) Differences in the methylation levels of CG sites between tumor and para-tumor in patients who were smokers. (F) Differences in the methylation levels of CG sites between tumor and para-tumor in patients who were non-smokers. (G) Association of the methylation levels of each CG site with TNFRSF10C expression. ${ }^{*} \mathrm{P}<0.05,{ }^{* *} \mathrm{P}<0.01,{ }^{* * *} \mathrm{P}<0.001,{ }^{* * * *} \mathrm{P}<0.0001$. F, forward primer; $\mathrm{R}$, reverse primer; TNFRSF10C, tumor necrosis factor receptor superfamily member 10c. 


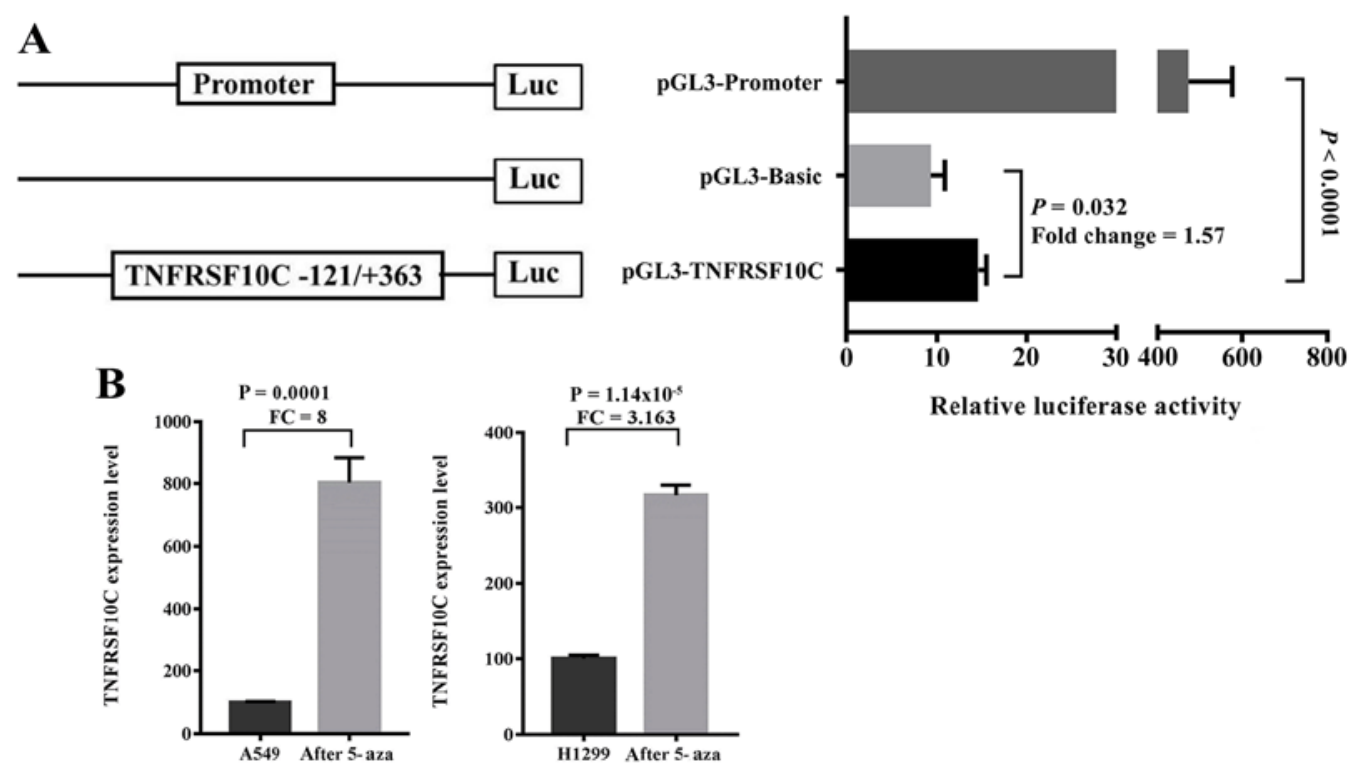

Figure 5. Dual luciferase assay to detect promoter activity of TNFRSF10C fragment in 293T cells. (A) pGL3-Promoter and pGL3-Basic plasmids were used as positive and baseline controls, respectively. (B) Lung cancer cell lines A549 and NCI-H1299 were treated with 5'-aza-deoxycytidine, and mRNA expression levels of TNFRSF10C were compared before and after treatment. 5-aza, 5'-aza-deoxycytidine; TNFRSF10C, tumor necrosis factor receptor superfamily member $10 \mathrm{c}$.

and patients who were non-smokers. TNFRSF10C methylation levels in tumor tissues of male patients were higher compared with those of female patients. These results demonstrated that TNFRSF10C methylation levels were associated with NSCLC.

Previous studies have reported that the relationship between TNFRSF10C gene hypermethylation and downregulated expression levels has been observed in human prostate cancer and pancreatic cancer $(27,29)$. In the present study, the levels of TNFRSF10C methylation were inversely correlated with mRNA expression levels in lung cancer tissue data from the cBioPortal database. 5'-Aza-deoxycytidine was used to treat two lung cancer cell lines, A549 and NCI-H1299, and the mRNA expression levels before and after treatment were compared. The results demonstrated that the mRNA expression levels of TNFRSF10C were significantly increased after 5'-aza-deoxycytidine-induced demethylation. These results indicated that the expression of TNFRSF10C was regulated by promoter methylation.

Hypermethylation of the TNFRSF10C promoter initiates the binding of TRAIL with tumor necrosis factors DR4 and DR5, and thus mediates the TRAIL-R signaling pathway (30). Although TRAIL-R induces apoptosis in a variety of cancer types, several types of tumor, such as cholangiocarcinoma and leukemia have recently been demonstrated to be resistant to TRAIL-R signaling $(31,32)$. In addition, TRAIL-R can activate the NF- $\kappa \mathrm{B}$ signaling pathway and promote the proliferation and migration of cancer cells, and thus TRAIL-R is associated with the development of cholangiocarcinoma and leukemia $(31,32)$.

Although smoking can cause epigenetic changes that induce cancer development, especially lung cancer, smoking is not the only cause of lung cancer (33). TRAIL-R has been demonstrated to promote the epidermal growth factor receptor (EGFR)-KRAS signaling pathway-mediated tumor development (34). A previous study reported that patients who were non-smokers harbored a higher frequency of EGFR mutations compared with smokers (35). Downregulation of TNFRSF10C expression via methylation promotes TRAIL-R deactivation in the EGFR-KRAS signaling pathway and promotes cancer progression and invasion (34). The results of the present study demonstrated that the TNFRSF10C methylation levels were higher in tumor tissues compared with distant non-tumor tissues in non-smokers; however, no significant differences were observed between the groups in smokers. These results may provide novel evidence supporting the epigenetic changes occurring in lung cancer in patients who are non-smokers.

The incidence of lung cancer in China is high in males at present (2). In the present study, the methylation levels of TNFRSF10C in tumor tissues from male patients were higher compared with distant non-tumor tissues, and the methylation levels in tumor tissues from male patients were significantly higher compared with those from female patients. These findings may provide novel evidence supporting the effects of sex on TNFRSF10C methylation levels in lung cancer.

TRAIL-R can mediate the inhibition of tumor development (36). TNFRSF10C and TNFRSF10D lack a cytoplasmic domain and do not contain transmembrane domains; thus, they are unable to participate in the transduction of apoptotic signals (37). Hypermethylation of TNFRSF10C promotes the binding of TRAIL to apoptotic receptors, thereby promoting apoptosis in cancer cells and preventing tumorigenesis (38). However, TNFRSF10D has a truncated death domain and hence is not capable of inducing apoptosis but protects against TRAIL-mediated apoptosis (39). The results of the present study indicated that TNFRSF10D methylation was not associated with lung cancer.

The present study primarily examined the methylation levels of the TNFRSF10C and TNFRSF10D genes in lung tumor and distant non-tumor tissues. The results demonstrated that TNFRSF10C hypermethylation was associated with NSCLC, especially in non-smokers and male patients. The 
underlying molecular mechanisms of the hypermethylation of TNFRSF10C in the development and progression of NSCLC need further investigation. DNA methylation of tissue biopsy may be used as a standard indicator of current pathological diagnosis $(40,41)$. However, tissue biopsy is inconvenient and traumatic. Liquid biopsy, such as circulating tumor cells, circulating tumor DNA and exosomes can provide more comprehensive disease information $(42,43)$. Furthermore, investigating the methylation levels of TNFRSF10C and TNFRSF10D genes of patients' blood samples, and combining data from both tissues and blood may allow their development as novel diagnostic biomarkers of lung cancer.

\section{Acknowledgements}

Not applicable.

\section{Funding}

This study was supported by The National Natural Science Foundation of China (grant no. 81773055), The Science Foundation of the Education Department of Fujian Province (grant no. JZ160438), the Key Talents Training Program of Fujian Provincial Health Commission (grant nos. 2017-ZQN-59 and 2017-ZQN-60), The Joint Funds for the Innovation Of Science And Technology of Fujian province (grant no. 2017Y9113) and The K.C. Wong Magna Fund in Ningbo University.

\section{Availability of data and materials}

The datasets used and/or analyzed during the present study are available from the corresponding author upon reasonable request.

\section{Authors' contributions}

YQ and LQ collected tissue samples and patient data, analyzed the data and wrote the manuscript. MQ and MZ carried out literature research and data interpretation. CY, JL, HL, ZZ and $\mathrm{CC}$ performed the experiments. SD designed the study and critically revised the manuscript for intellectually important content. All authors have read and approved the final manuscript.

\section{Ethics approval and consent to participate}

The present study was approved by The Ethics Committee of the First Affiliated Hospital of Fujian Medical University (Fuzhou, China; approval number: 2017-KY-068). All patients provided written informed consent for participation in this study.

\section{Patient consent for publication}

Not applicable.

\section{Competing interests}

The authors declare that they have no competing interests.

\section{References}

1. Torre LA, Bray F, Siege RL, Ferlay J, Lortet-Tieulent J and Jemal A: Global cancer statistics, 2012. CA Cancer J Clin 65: 87-108, 2015.

2. Chen W, Zheng R, Baade PD, Zhang S, Zeng H, Bray F, Jemal A Yu XQ and He J: Cancer statistics in China, 2015. CA Cancer J Clin 66: 115-132, 2016.

3. Inage T, Nakajima T, Yoshino I and Yasufuku K: Early lung cancer detection. Clin Chest Med 39: 45-55, 2018.

4. NSCLC Meta-analysis Collaborative Group: Preoperative chemotherapy for non-small-cell lung cancer: A systematic review and meta-analysis of individual participant data. Lancet 383: 1561-1571, 2014.

5. Le Chevalier T: Adjuvant chemotherapy for resectable non-small-cell lung cancer: Where is it going? Ann Oncol 21 (Suppl 7): vii196-vii198, 2010.

6. Ji P, Ding D, Qin N, Wang C, Zhu M, Li Y, Dai J, Jin G, Hu Z, Shen $\mathrm{H}$, et al: Systematic analyses of genetic variants in chromatin interaction regions identified four novel lung cancer susceptibility loci. J Cancer 11: 1075-1081, 2020

7. Tang D, Zhao YC, Liu H, Luo S, Clarke JM, Glass C, Su L, Shen S, Christiani DC, Gao W and Wei Q: Potentially functional genetic variants in PLIN2, SULT2A1 and UGT1A9 genes of the ketone pathway and survival of nonsmall cell lung cancer. Int J Cancer: Feb 18, 2020 (Epub ahead of print).

8. Jakopovic M, Thomas A, Balasubramaniam S, Schrump D, Giaccone $\mathrm{G}$ and Bates SE: Targeting the epigenome in lung cancer: Expanding approaches to epigenetic therapy. Front Oncol 3: 261, 2013.

9. Berger SL, Kouzarides T, Shiekhattar R and Shilatifard A: An operational definition of epigenetics. Genes Dev 23: 781-783, 2009.

10. Rana AK and Ankri S: Reviving the RNA world: An insight into the appearance of RNA Methyltransferases. Front Genet 7: 99, 2016.

11. Rauch T, Wang Z, Zhang X, Zhong X, Wu X, Lau SK, Kernstine KH, Riggs AD and Pfeifer GP: Homeobox gene methylation in lung cancer studied by genome-wide analysis with a microarray-based methylated $\mathrm{CpG}$ island recovery assay. Proc Natl Acad Sci USA 104: 5527-5532, 2007.

12. Castro M, Grau L, Puerta P, Gimenez L, Venditti J, Quadrelli S and Sánchez-Carbayo M: Multiplexed methylation profiles of tumor suppressor genes and clinical outcome in lung cancer. J Transl Med 8: 86, 2010.

13. Emery JG, McDonnellP, Burke MB, Deen KC, Lyn S, Silverman C, Dul E, Appelbaum ER, Eichman C, DiPrinzio R, et al: Osteoprotegerin is a receptor for the cytotoxic ligand TRAIL. J Biol Chem 273: 14363-14367, 1998.

14. Allen JE, Krigsfeld G, Mayes PA, Patel L, Dicker DT, Patel AS, Dolloff NG, Messaris E, Scata KA, Wang W, et al: Dual inactivation of Akt and ERK by TIC10 signals Foxo3a nuclear translocation, TRAIL gene induction, and potent antitumor effects. Sci Transl Med 5: 171ra17, 2013.

15. Dimberg LY, Anderson CK, Camidge R, Behbakht K, Thorburn A and Ford HL: On the TRAIL to successful cancer therapy? Predicting and counteracting resistance against TRAIL-based therapeutics. Oncogene 32: 1341-1350, 2013.

16. Lim B, Allen JE, Prabhu VV, Talekar MK, Finnberg NK and El-Deiry WS: Targeting TRAIL in the treatment of cancer: New developments. Expert Opin Ther Targets 19: 1171-1185, 2015.

17. Soria JC, Smit E, Khayat D, Besse B, Yang X, Hsu CP, Reese D, Wiezorek $J$ and Blackhall $F$ : Phase $1 \mathrm{~b}$ study of dulanermin (recombinant human Apo2L/TRAIL) in combination with paclitaxel, carboplatin, and bevacizumab in patients with advanced non-squamous non-small-cell lung cancer. J Clin Oncol 28: 1527-1533, 2010.

18. Wainberg ZA, Messersmith WA, Peddi PF, Kapp AV, Ashkenazi A, Royer-Joo S, Portera CC and Kozloff MF: A phase 1B study of dulanermin in combination with modified FOLFOX6 plus bevacizumab in patients with metastatic colorectal cancer. Clin Colorectal Cancer 12: 248-254, 2013.

19. Cheah CY, Belada D, Fanale MA, Janikova A, Czucman MS, Flinn IW, Kapp AV, Ashkenazi A, Kelley S, Bray GL, et al: Dulanermin with rituximab in patients with relapsed indolent B-cell lymphoma: An open-label phase 1b/2 randomised study. Lancet Haematol 2: e166-e174, 2015. 
20. Herbst RS, Eckhardt SG, Kurzrock R, Ebbinghaus S, O'Dwyer PJ, Gordon MS, Novotny W, Goldwasser MA, Tohnya TM, Lum BL, et al: Phase I dose-escalation study of recombinant human Apo2L/TRAIL, a dual proapoptotic receptor agonist, in patients with advanced cancer. J Clin Oncol 28: 2839-2846, 2010.

21. Braithwaite AT, Marriott HM and Lawrie A: Divergent roles for TRAIL in lung diseases. Front Med (Lausanne) 5: 212, 2018

22. Tessema M, Yu YY, Stidley CA, Machida EO, Schuebel KE, Baylin SB and Belinsky SA: Concomitant promoter methylation of multiple genes in lung adenocarcinomas from current, former and never smokers. Carcinogenesis 30: 1132-1138, 2009.

23. Raff T, van der Giet M, Endemann D, Wiederholt T and Paul M: Design and testing of beta-actin primers for RT-PCR that do not co-amplify processed pseudogenes. Biotechniques 23: 456-460, 1997.

24. Kristensen LS, Mikeska T, Krypuy M and Dobrovic A: Sensitive melting analysis after real time-methylation specific PCR (SMART-MSP): High-throughput and probe-free quantitative DNA methylation detection. Nucleic Acids Res 36: e42, 2008.

25. Livak KJ and Schmittgen TD: Analysis of relative gene expression data using real-time quantitative PCR and the 2(-Delta Delta C(T)) method. Methods 25: 402-408, 2001.

26. Vaitkienè $P$, Skiriute $D$, Skauminas $K$ and Tamašauskas $A$ : GATA4 and DcR1 methylation in glioblastomas. Diagn Pathol 8: 7,2013.

27. Cheng Y, Kim JW, Liu W, Dunn TA, Luo J, Loza MJ, Kim ST, Zheng SL, Xu J, Isaacs WB and Chang BL: Genetic and epigenetic inactivation of TNFRSF10C in human prostate cancer. Prostate 69: 327-335, 2009.

28. Tserga A, Michalopoulos NV, Levidou G, Korkolopoulou P, Zografos G, Patsouris E and Saetta AA: Association of aberrant DNA methylation with clinicopathological features in breast cancer. Oncol Rep 27: 1630-1638, 2012.

29. Cai HH, Sun YM, Miao Y, Gao WT, Peng Q, Yao J and Zhao HL: Aberrant methylation frequency of TNFRSF10C promoter in pancreatic cancer cell lines. Hepatobiliary Pancreat Dis Int 10: 95-100, 2011

30. von Karstedt S, Montinaro A and Walczak H: Exploring the TRAILs less travelled: TRAIL in cancer biology and therapy. Nat Rev Cancer 17: 352-366, 2017.

31. Ishimura N, Isomoto H, Bronk SF and Gores GJ: Trail induces cell migration and invasion in apoptosis-resistant cholangiocarcinoma cells. Am J Physiol Gastrointest Liver Physiol 290: G129-G136, 2006.

32. Ehrhardt H, Fulda S, Schmid I, Hiscott J, Debatin KM and Jeremias I: TRAIL induced survival and proliferation in cancer cells resistant towards TRAIL-induced apoptosis mediated by NF-kappaB. Oncogene 22: 3842-3852, 2003.
33. Vaz M,Hwang SY,Kagiampakis I,Phallen J,Patil A,O'Hagan HM, Murphy L, Zahnow CA, Gabrielson E, Velculescu VE, et al: Chronic cigarette smoke-induced epigenomic changes precede sensitization of bronchial epithelial cells to single-step transformation by KRAS mutations. Cancer Cell 32: 360-376.e6, 2017.

34. von Karstedt S, Conti A, Nobis M, Montinaro A, Hartwig T, Lemke J, Legler K, Annewanter F, Campbell AD, Taraborrelli L, et al: Cancer cell-autonomous TRAIL-R signaling promotes KRAS-driven cancer progression, invasion, and metastasis. Cancer Cell 27: 561-573, 2015.

35. Chapman AM, Sun KY, Ruestow P, Cowan DM and Madl AK: Lung cancer mutation profile of EGFR, ALK, and KRAS: Meta-analysis and comparison of never and ever smokers. Lung Cancer 102: 122-134, 2016.

36. Finnberg N, Klein-Szanto AJ and El-Deiry WS: TRAIL-R deficiency in mice promotes susceptibility to chronic inflammation and tumorigenesis. J Clin Invest 118: 111-123, 2008.

37. Shivapurkar N, Toyooka S, Toyooka KO, Reddy J, Miyajima K, Suzuki M, Shigematsu H, Takahashi T, Parikh G, Pass HI, et al: Aberrant methylation of trail decoy receptor genes is frequent in multiple tumor types. Int J Cancer 109: 786-792, 2004.

38. van Noesel MM, van Bezouw S, Salomons GS, Voûte PA, Pieters R, Baylin SB, Herman JG and Versteeg R: Tumor-specific down-regulation of the tumor necrosis factor-related apoptosis-inducing ligand decoy receptors DcR1 and DcR2 is associated with dense promoter hypermethylation. Cancer Res 62: 2157-2161, 2002.

39. Pan G, Ni J, Yu G, Wei YF and Dixit VM: TRUNDD, a new member of the TRAIL receptor family that antagonizes TRAIL signalling. FEBS Lett 424: 41-45, 1998.

40. Diaz-Lagares A, Mendez-Gonzalez J, Hervas D, Saigi M, Pajares MJ, Garcia D, Crujerias AB, Pio R, Montuenga LM, Zulueta J, et al: A novel epigenetic signature for early diagnosis in lung cancer. Clin Cancer Res 22: 3361-3371, 2016.

41. Dong S, Li W, Wang L, Hu J, Song Y, Zhang B, Ren X, Ji S, Li J, Xu P, et al: Histone-related genes are hypermethylated in lung cancer and hypermethylated HIST1H4F could serve as a pan-cancer biomarker. Cancer Res 79: 6101-6112, 2019.

42. Constâncio V, Nunes SP, Henrique R and Jerónimo C: DNA methylation-based testing in liquid biopsies as detection and prognostic biomarkers for the four major cancer types. Cells 9: pii: E624, 2020.

43. Nunes SP, Diniz F, Moreira-Barbosa C, Constâncio V, Silva AV, Oliveira J, Soares M, Paulino S, Cunha AL, Rodrigues J, et al: Subtyping lung cancer using DNA methylation in liquid biopsies. J Clin Med 8: pii: E1500, 2019.

This work is licensed under a Creative Commons Attribution-NonCommercial-NoDerivatives 4.0 International (CC BY-NC-ND 4.0) License. 